\title{
IncRNA SNHG5 promotes cell proliferation, migration and invasion in oral squamous cell carcinoma by sponging miR-655-3p/FZD4 axis
}

\author{
LIJIANG YU $^{1 *}$, LINGLI HUO $^{2 *}$, XIAOLIN SHAO ${ }^{3}$ and JIZHI ZHAO ${ }^{1}$ \\ ${ }^{1}$ Department of Oral and Maxillofacial Surgery, Peking Union Medical College Hospital, \\ Chinese Academy of Medical Sciences, Beijing 100730; ${ }^{2}$ Department of Stomatology, \\ Traditional Chinese Medicine Hospital of Shijingshan District, Beijing 100043; ${ }^{3}$ Department of Stomatology, \\ Beijing Ditan Hospital, Capital Medical University, Beijing 100013, P.R. China
}

Received April 13, 2020; Accepted September 29, 2020

DOI: $10.3892 / \mathrm{ol} .2020 .12173$

\begin{abstract}
Recently, previous studies have shown that long non-coding RNA (lncRNA) can act as a tumor promoter or inhibitor in the pathogenesis of oral squamous cell carcinoma (OSCC). However, the regulatory mechanism of lncRNA SNHG5 is unknown in OSCC. Therefore, the functional mechanism of lncRNA SNHG5 in OSCC was initially revealed in this study. Here, RT-qPCR and western blot analysis were used to assess mRNA and protein expression. The functional mechanism of SNHG5 was investigated by MTT, Transwell and luciferase reporter assays. The results showed that SNHG5 expression was upregulated in OSCC and promoted the viability, migration and invasion of OSCC cells. In addition, SNHG5 is the sponge of miR-655-3p in OSCC. And miR-655-3p was found to play an inhibitory effect in OSCC by interacting with SNHG5. Moreover, miR-655-3p directly targets FZD4 and negatively regulates its expression in OSCC. Functionally, FZD4 promoted the progression of OSCC by interacting with the SNHG5/miR-655-3p axis. In conclusion, lncRNA SNHG5 promotes cell proliferation, migration and invasion in OSCC by regulating miR-655-3p/FZD4 axis.
\end{abstract}

\section{Introduction}

Oral cancer is one of the most common head and neck malignancies, while oral squamous cell carcinoma (OSCC) is the most common (1). It has been reported that oral cancer accounts

Correspondence to: Dr Jizhi Zhao, Department of Oral and Maxillofacial Surgery, Peking Union Medical College Hospital, Chinese Academy of Medical Sciences, 1 Shuai Fu Yuan, Dongcheng, Beijing 100730, P.R. China

E-mail: sumwkb6241@163.com

*Contributed equally

Key words: SNHG5, oral squamous cell carcinoma, miR-655-3p, FZD4 for 1.9-3.5\% of systemic malignancies. In addition, it accounts for $4.7-20.3 \%$ of head and neck malignancies, second only to nasopharyngeal carcinoma (2). Today, surgical resection and radiotherapy are still the two most effective methods for the treatment of OSCC (3). In addition, combined treatment with the poly(ADP) ribose polymerase inhibitors $1 / 2$ (PARP1/2) is also a valid therapeutic strategy for OSCC (4). The overall 5-year survival rate of OSCC patients is $50-70 \%$. The 5-year survival rate at the stage I can be as high as $90 \%$ or higher, while the 5-year survival rate at stage IV is only $\sim 10 \%$. The main reason for the failure of OSCC treatment is local recurrence of the primary tumor (5). Therefore, early diagnosis and treatment are the keys to the treatment of OSCC.

Long non-coding RNA (lncRNA) is a type of RNA molecule whose transcript length exceeds $200 \mathrm{nt}$. IncRNA does not encode protein, but regulates the expression level of genes in the form of RNA at multiple levels (epigenetic regulation, transcription regulation, and post-transcriptional regulation) (6). In addition, IncRNAs regulate the tumorigenesis and development of cancers by affecting some important cellular biological activities, such as cell viability, differentiation, motility and apoptosis (7). Previous studies have shown that lncRNAs can serve as tumor suppressors or oncogenes in OSCC, such as lncRNA MORT and CASC9 $(8,9)$. More specifically, overexpression of lncRNA MORT restrained cell proliferation in OSCC by downregulating ROCK1 (8). In addition, the increased expression of IncRNA CASC9 promoted tumor progression through the AKT/mTOR pathway in OSCC (9). Recently, the different roles of 1ncRNA SNHG5 in malignant tumors have attracted our attention. Low expression of SNHG5 was found in gastric cancer, and overexpression of SNHG5 suppressed the progression of gastric cancer (10). However, SNHG5 was upregulated in glioma, and knockdown of SNHG5 inhibited the malignant cellular phenotype of glioma (11). These results prove that SNHG5 may have tissue specificity. However, the regulatory mechanism of SNHG5 in OSCC is still unknown.

In addition, SNHG5 is predicted to have a binding site with miR-655-3p. Meanwhile, the abnormal expression and function of miR-655-3p have been investigated in other cancers. Downregulation of miR-655-3p has been detected 
in retinoblastoma and esophageal squamous cell carcinoma $(12,13)$. Functionally, miR-655-3p was found to inhibit cell proliferation in human lip cells (14). In addition, miR-655-3p restrained the migration and invasion of non-small cell lung cancer cells by targeting PTTG1 (15). Here, Frizzled-4 (FZD4) is predicted as the target of miR-655-3p. In addition, abnormal expression of FZD4 has been identified in human cancers. For example, FZD4 was abnormally upregulated and acted as an oncogene in lung cancer and prostate cancer $(16,17)$. In addition, miR-101 has been found to suppress the migration and invasion of bladder cancer cells by targeting FZD4 (18). However, the functional mechanism of miR-655-3p/FZD4 axis in OSCC is unclear. Besides, lncRNA HOXD-AS1 was found to promote cell proliferation, migration and invasion in ovarian cancer through the miR-608/FZD4 axis (19). However, it is unclear how lncRNA SNHG5 regulates OSCC progression by interacting with the miR-655-3p/FZD4 axis.

Therefore, the functions of lncRNA SNHG5, miR-655-3p and FZD4 as well as their possible mechanisms were investigated in OSCC. This research will help us better understand the pathogenesis of OSCC and provide possible biomarkers for OSCC treatment.

\section{Materials and methods}

Clinical tissues. Forty-two OSCC tissues and paired normal tissues were obtained from Peking Union Medical College Hospital. The informed consents of OSCC patients were collected before the experiment. All OSCC patients have not received chemotherapy or radiotherapy before undergoing surgery. This study was approved by the Institutional Ethics Committee of Peking Union Medical College Hospital, approval number was 2017PUMC26. All patients provided written informed consents.

Cell lines and culture. OSCC cell line SCC-4 (ZKCC-X1937) and Immortalized Human Oral Mucosal Epithelial Cells hTERT-OME (ATCC ${ }^{\circledR}$ PCS-200-014 ${ }^{\mathrm{TM}}$ ) were obtained from Beijing Zhongke Quality Inspection Biotechnology Co., Ltd. and American Type Culture Collection (ATCC). These cells were cultured in Dulbecco's modified Eagle's medium (DMEM) containing 10\% FBS and cultured in an incubator with $5 \% \mathrm{CO}_{2}$ at $37^{\circ} \mathrm{C}$.

Cell transfection. SNHG5 and FZD4 overexpression vectors or siRNAs, and miR-655-3p mimics(B01001)orinhibitor(B03001) were obtained from GenePharma. For transfection, cells were seeded into 6 -well plates at a density of $2 \times 10^{5}$ cells/well. Cells were transfected with miR-655-3p mimics or inhibitor (100 pmol), SNHG5 and FZD4 siRNA (100 pmol) or vectors $(4 \mu \mathrm{g})$ using Lipofectamine ${ }^{\circledR} 2000$ (Invitrogen; Thermo Fisher Scientific, Inc.) when $60-70 \%$ confluence was achieved, in accordance with the manufacturer's protocol. Subsequent to transfection for 6-8 h, cell culture medium was replaced with DMEM without antibiotics and incubated at $37^{\circ} \mathrm{C}$ with $5 \% \mathrm{CO}_{2}$.

$R N A$ isolation, reverse transcription and $R T-q P C R$. TRIzol reagent (Invitrogen; Thermo Fisher Scientific, Inc.) was used to extract total RNA. RNA was reversely transcribed to complementary DNA (cDNA) using a Reverse Transcription Kit (Takara). RT-qPCR assay was performed using Real-time PCR
Mixture assays (Takara) and corresponding primers. SNHG5 and miR-655-3p expression were normalized to U6, when FZD4 was normalized to GAPDH. Their expressions were quantified by the $2^{-\Delta \Delta c q}$ method. The primers used were: SNHG5 forward 5'-CGAGTAGCCAGTGAAGATAATG-3'; SNHG5 reverse 5'-CACACAACAGTCAAGTAAACC-3'; miR-655-3p forward: 5'-CAATCCTTACTCCAGCCAC-3' and reverse, 5'-GTGTCT TAAGGCTAGGCCTA-3'; U6-forward: 5AAAT-3' and reverse, 5'-TTGCGTGTCAT-3'; FZD4-forward: 5'-GGTGGCTCCCCT CTTTACTT-3' and reverse, 5'-ATCACACACGTTGCAGAA C-3'; GAPDH forward: 5'-ACAACTTTGGTATCGTGGAAG G-3', and reverse, 5'-GCCATCACGCCACAGTTTC-3'.

MTT assay. Prepared transfected SCC- 4 cells $\left(2 \times 10^{3}\right.$ cells/well) were put in a 96-well plate. Next, the SCC-4 cells were incubated in DMEM medium for 24, 48, 72 or $96 \mathrm{~h}$. Then, the cells were incubated with $10 \mu \mathrm{l}$ MTT solution for $4 \mathrm{~h}$. MTT solution was then aspirated. And Formazan solution was added to completely dissolve the crystals. The absorbance at $490 \mathrm{~nm}$ was examined with a microplate reader (Olympus Corp.).

Transwellassay.Matrigelmatrix (1:8;50 $\mu 1 /$ well;BDBiosciences) in the upper chamber was used to detect cell invasion. After $30 \mathrm{~min}$, the SCC- 4 cell suspension $\left(2 \times 10^{3}\right.$ cells/well) was added to the Transwell upper chamber. The lower chamber was added with DMEM medium (10\% FBS). After $48 \mathrm{~h}$ of incubation, crystal violet (Beyotime Institute of Biotechnology) was used to fix and stain the cells on the lower surface of the membrane for $30 \mathrm{~min}$. Cell migration assay was performed without Matrigel. The other steps are the same as cell invasion. An optical microscope was used to count the number of stained cells for five randomly selected fields.

Dual luciferase reporter assay. The 3'-UTR of wild-type and mutant SNHG5 (wt-SNHG5 and mut-SNHG5) or FZD4 (wt-FZD4 and mut-FZD4) were inserted into the pmiR-GLO vectors (Promega Corporation). The above reporter plasmids and miR-655-3p mimics were transfected into SCC-4 cells. After $48 \mathrm{~h}$, the activity of firefly and Renilla luciferase was measured by the dual-luciferase reporter gene system (Promega Corporation).

Western blot analysis. Protein samples were extracted by RIPA lysis buffer (Beyotime Institute of Biotechnology). Protein concentration was measured using Bicinchoninic Acid protein assay kit (Sigma-Aldrich; Merck KGaA). Next, $25 \mu \mathrm{g}$ protein was separated by $10 \%$ SDS-PAGE and transferred to PVDF membranes. And the protein was blocked at room temperature for $2 \mathrm{~h}$ with $5 \%$ non-fat milk. Then, protein samples were incubated with FZD4 (ab83042, 1:1,000 dilution, Abcam, rabbit polyclonal antibodies) and GAPDH (ab8245, 1:1,000 dilution, Abcam, mouse monoclonal antibody) primary antibodies overnight at $4^{\circ} \mathrm{C}$. After washing with TBST, protein samples were incubated with corresponding horseradish peroxidase-conjugated secondary antibodies (ab205719, 1:5,000 dilution; Abcam). Protein bands were visualized by ECL kit (Beyotime Institute of Biotechnology) and were quantified with Image Lab Software (Bio-Rad Laboratories, Inc.).

Statistical analysis. All statistical analyses were conducted by using SPSS 19.0 or Graphpad Prism 6. All data were presented 

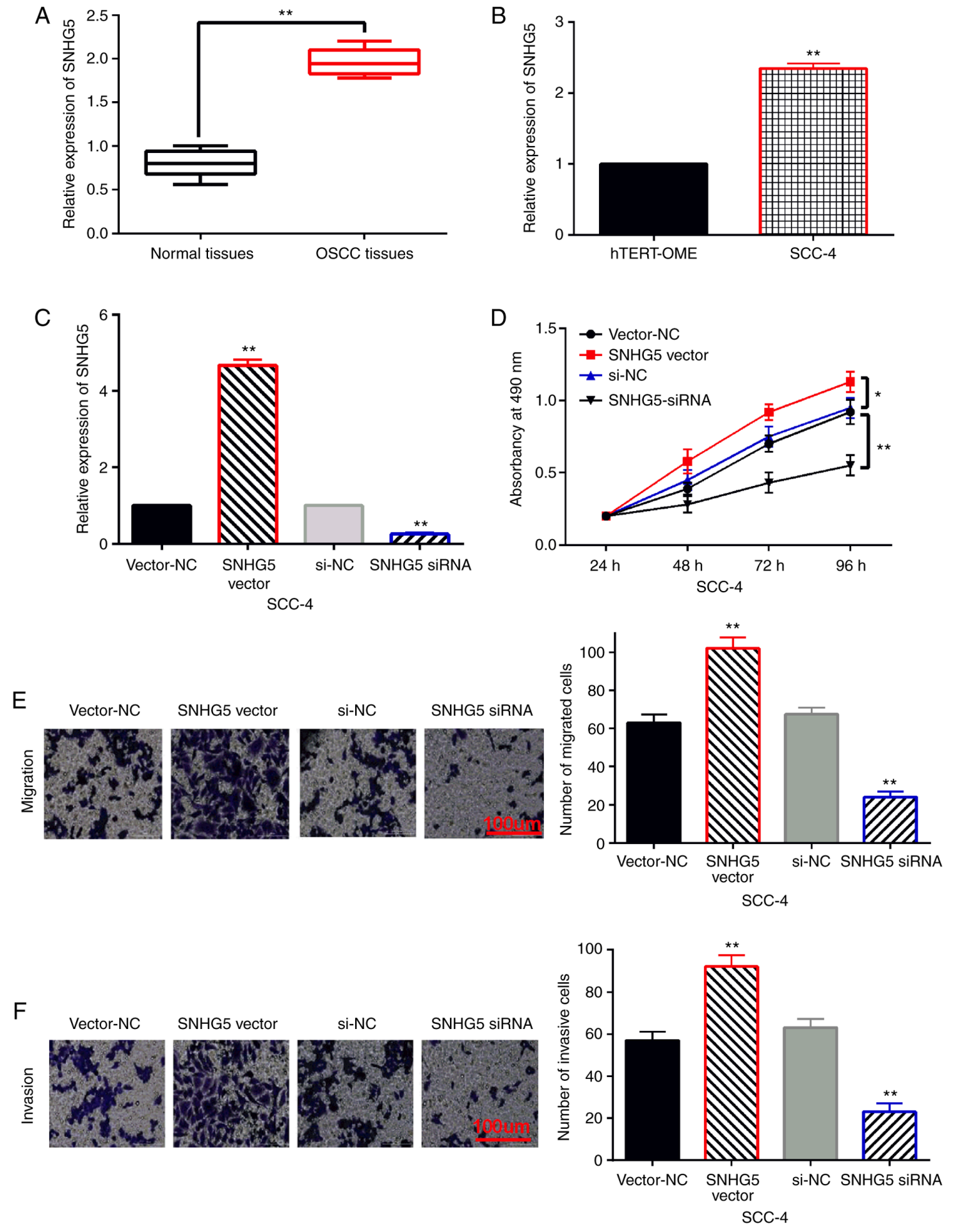

Figure 1. The dysfunction of lncRNA SNHG5 is identified in OSCC. (A) The mRNA SNHG5 expression in OSCC tissues (N=42) (B) SNHG5 expression in SCC-4 and hTERT-OME cells (C) SNHG5 expression in SCC-4 cells with SNHG5 siRNA or overexpression vector. (D) Cell proliferation in SCC-4 cells with SNHG5 siRNA or overexpression vector (E and F) Cell migration and invasion in SCC-4 cells with SNHG5 siRNA or overexpression vector ${ }^{*} \mathrm{P}<0.05,{ }^{* *} \mathrm{P}<0.01$. lncRNA, long non-coding RNA; OSCC, oral squamous cell carcinoma.

as the mean \pm standard deviation from at least three separate experiments. Differences between two groups were estimated by unpaired two-tailed Student t-test. Differences between multiple groups were compared using one-way analysis of variance followed by Tukey's post hoc test. The correlations between miR-655-3p expression and IncRNA SNHG5 or FZD4 expression in OSCC tissues were analyzed using Spearman's rank test. $\mathrm{P}<0.05$ indicates a statistically significant difference.

\section{Results}

Upregulation of IncRNA SNHG5 promotes cell proliferation, migration and invasion in OSCC. The role of lncRNA SNHG5 was explored in OSCC. First, SNHG5 expression was detected in OSCC tissues and cells. Compared to normal tissues, the expression of SNHG5 in OSCC tissues was increased (Fig. 1A). In addition, higher expression of 


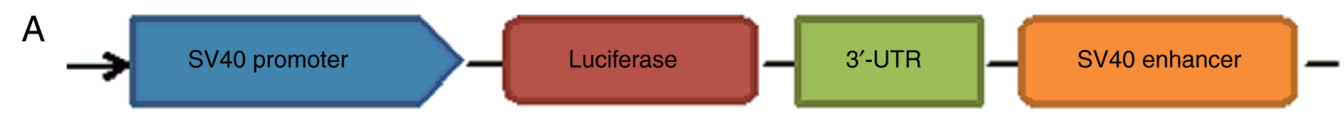

SNHG5-WT 5'-ACAGAAGUGCAGUUUAAUGUAUUAU-3'

Has-miR-655-3p

SNHG5-Mut

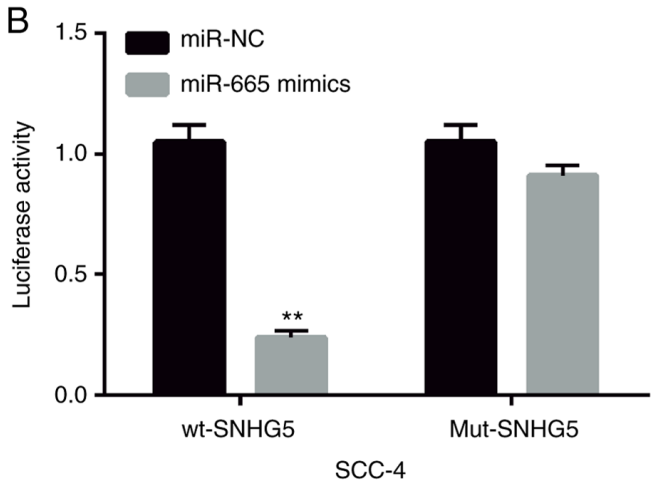

D

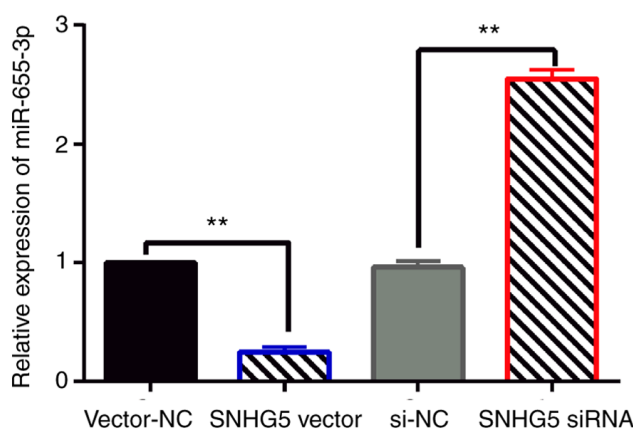

SCC-4
3'-UUUCUCCAAUUGGUACAUAAUA-5'

5'-ACAGAAGUGCAGUUUAUACAUAAUU-3'

C

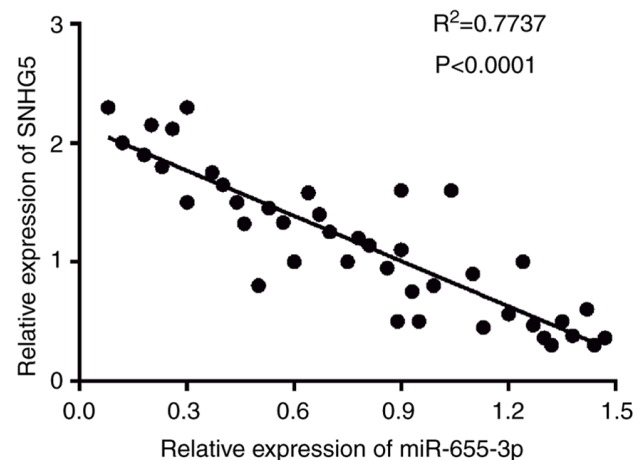

E

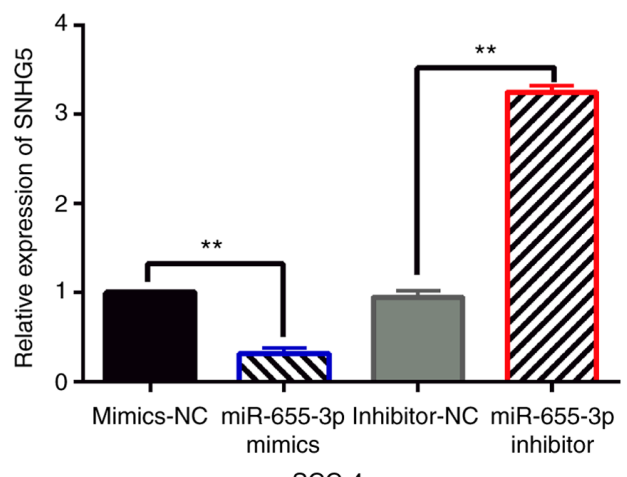

SCC-4

Figure 2. IncRNA SNHG5 acts as a molecular sponge of miR-655-3p in OSCC. (A) The binding sites between SNHG5 with miR-655-3p. (B) Luciferase reporter assay was employed to identify the binding site between miR-655-3p and the 3'-UTR of SNHG5. (C) miR-655-3p was negatively correlated with SNHG5 in OSCC tissues. (D) miR-655-3p expression regulated by SNHG5 siRNA or vector in SCC-4 cells (E) SNHG5 expression in SCC-4 cells containing miR-655-3p mimics or inhibitor ${ }^{* *} \mathrm{P}<0.01$. IncRNA, long non-coding RNA; OSCC, oral squamous cell carcinoma.

SNHG5 was also found in SCC-4 cells than that in the hTERT-OME cells (Fig. 1B). Next, SNHG5 siRNA or overexpression vector was transfected into SCC-4 cells. We found that the expression of SNHG5 was reduced by its siRNA and was enhanced by its overexpression vector in SCC-4 cells (Fig. 1C). In addition, upregulation of SNHG5 promoted cell proliferation, while knockdown of SNHG5 restrained cell proliferation in SCC-4 cells (Fig. 1D). Meanwhile, SCC-4 cell migration was promoted by SNHG5 overexpression and was inhibited by SNHG5 downregulation (Fig. 1E). The same effect of SNHG5 on cell invasion was also examined in SCC-4 cells (Fig. 1F). Briefly, upregulation of lncRNA SNHG5 promotes cell proliferation, migration and invasion in OSCC.

lncRNA SNHG5 acts as a molecular sponge of miR-655-3p in OSCC. IncRNA SNHG5 was found to have a binding site with miR-655-3p in the starBase database (http://starbase.sysu. edu.cn/, Fig. 2A). Dual luciferase reporter was used to verify the relationship between them. It was found that miR-655-3p mimics reduced the luciferase activity of wt-SNHG5, but had little effect on mut-SNHG5 luciferase activity in SCC-4 cells (Fig. 2B). In addition, we found that SNHG5 was negatively correlated with the expression of miR-655-3p in OSCC tissues (Fig. 2C). Next, SNHG5 siRNA or vector and miR-655-3p mimics or inhibitor were transfected into SCC-4 cells, respectively. RT-qPCR showed that miR-655-3p expression was reduced by SNHG5 upregulation and enhanced by SNHG5 downregulation in SCC-4 cells (Fig. 2D). Meanwhile, miR-655-3p mimics reduced SNHG5 expression, while miR-655-3p inhibitor promoted SNHG5 expression in SCC-4 cells (Fig. 2E). These results indicate that SNHG5 is a competitive miRNA of miR-655-3p in OSCC.

miR-655-3p is involved in OSCC progression by mediating lncRNA SNHG5. Next, the abnormal expression and function of miR-655-3p was investigated in OSCC development. Compared to normal tissues, miR-655-3p expression in 

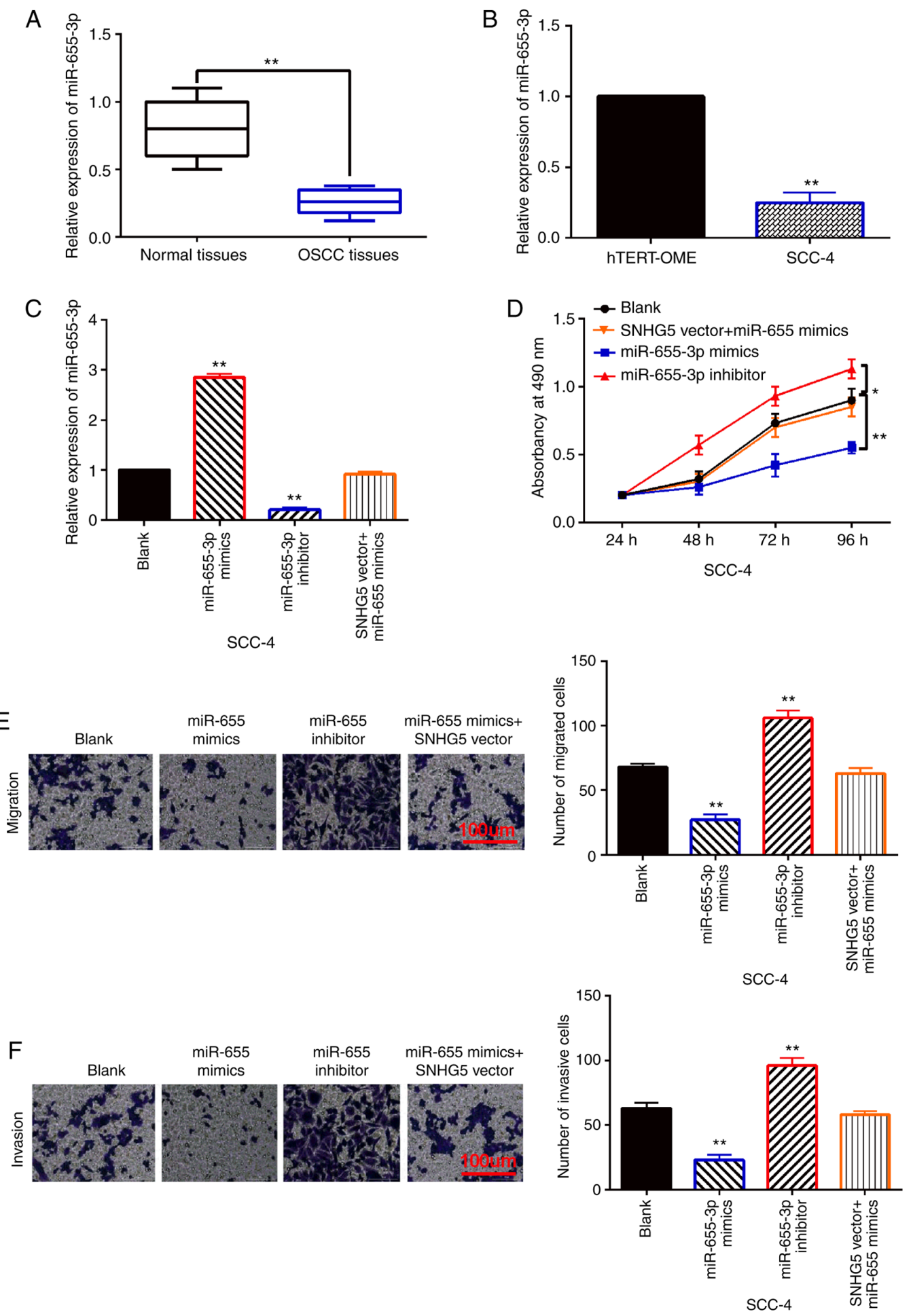

Figure 3. The dysregulation of miR-655-3p is involved in OSCC progression by mediating lncRNA SNHG5. (A) The mRNA miR-655-3p expression in OSCC tissues (B) miR-655-3p expression in SCC-4 and hTERT-OME cells (C) miR-655-3p expression in SCC-4 cells with miR-655-3p mimics and inhibitor or SNHG5 vector. (D) Cell proliferation in SCC-4 cells with miR-655-3p mimics and inhibitor or SNHG5 vector. (E and F) Cell migration and invasion in SCC-4 cells with miR-655-3p mimics and inhibitor or SNHG5 vector " $\mathrm{P}<0.05,{ }^{* * *} \mathrm{P}<0.01$. OSCC, oral squamous cell carcinoma; lncRNA, long non-coding RNA.

OSCC tissues was found to be downregulated (Fig. 3A). And compared with hTERT-OME cells, downregulation of miR-655-3p was detected in SCC-4 cells (Fig. 3B). To explore the function of miR-655-3p in OSCC, miR-655-3p mimics or inhibitor were transfected into SCC-4 cells. RT-qPCR showed that miR-655-3p mimics increased its expression, while miR-655-3p inhibitor reduced its expression in SCC-4 cells
(Fig. 3C). In addition, this increased expression of miR-655-3p was reduced by SNHG5 upregulation (Fig. 3C). More importantly, cell proliferation was restrained by miR-655-3p overexpression and promoted by miR-655-3p downregulation in SCC-4 cells (Fig. 3D). Similarly, miR-655-3p mimics also inhibited SCC-4 cell migration and invasion, miR-655-3p inhibitors also promoted the migration and invasion of SCC-4 


\section{A}

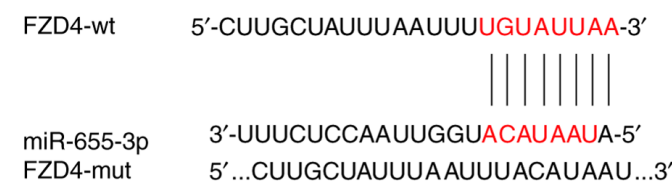

C

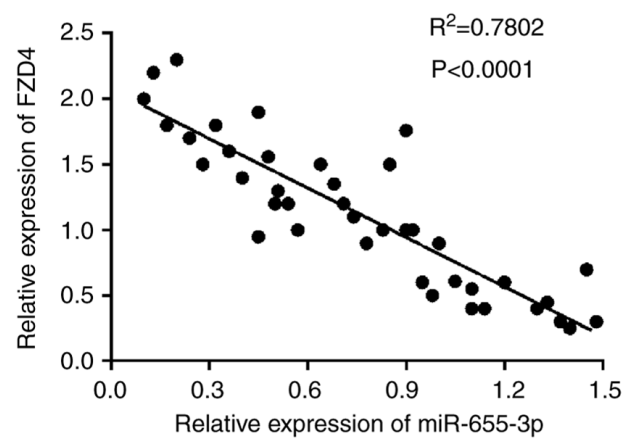

E

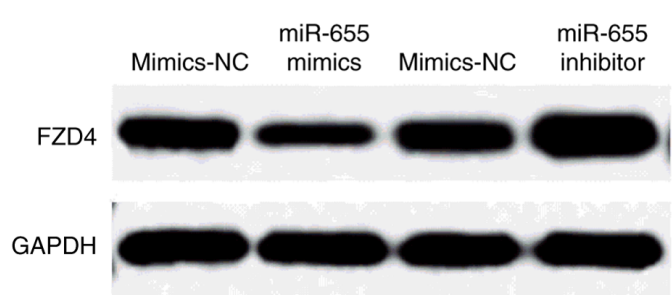

B

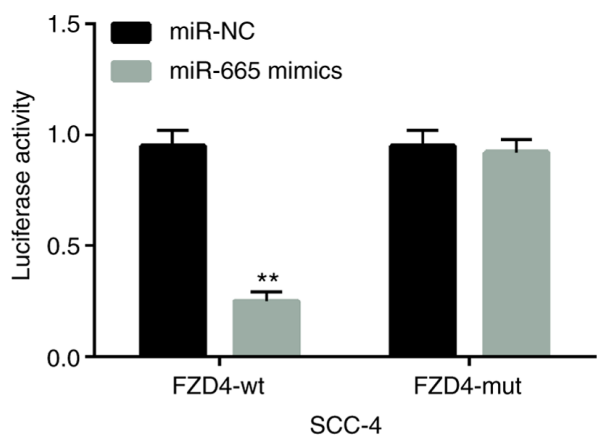

D

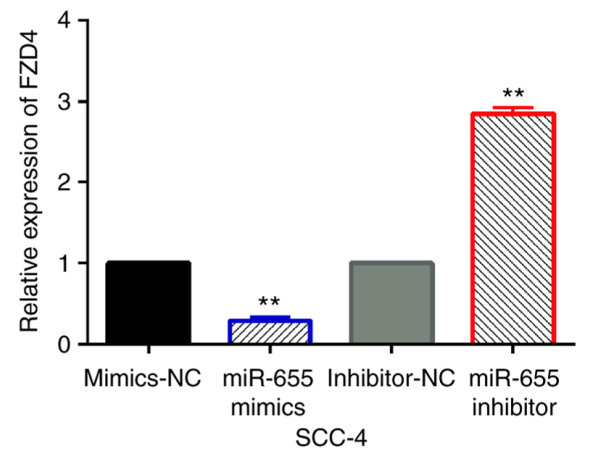

F

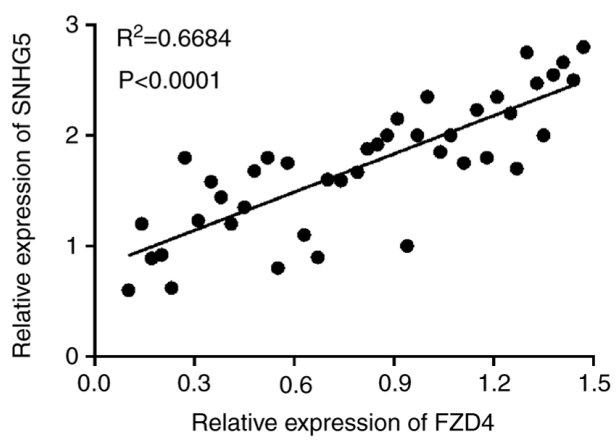

Figure 4. FZD4 is a direct target of miR-655-3p. (A) The binding sites between FZD4 and miR-655-3p. (B) Luciferase reporter assay was employed to identify the binding site between miR-655-3p and the 3'-UTR of FZD4. (C) miR-655-3p was negatively correlated with FZD4 in OSCC tissues. (D and E) FZD4 expression regulated by miR-655-3p mimics or inhibitor in SCC-4 cells (F) SNHG5 was positively correlated with FZD4 in OSCC tissues. ${ }^{* *} \mathrm{P}<0.01$. OSCC, oral squamous cell carcinoma.

cells (Fig. 3E and F). In addition, upregulation of SNHG5 weakened the inhibitory effect of miR-655-3p on cell proliferation, migration and invasion in SCC-4 cells (Fig. 3D-F). In conclusion, miR-655-3p acts as a tumor suppressor in OSCC by competitively binding to SNHG5.

FZD4 is a direct target of miR-655-3p. Then, the TargetScan database (http://www.targetscan.org) predicts that FZD4 is the target of miR-655-3p (Fig. 4A). Luciferase reporter assay was performed to verify the prediction. It was found that miR-214-3p mimics reduced the luciferase activity of wt-FZD4, but had no effect on mut-FZD4 luciferase activity (Fig. 4B). In addition, FZD4 was negatively correlated with the expression of miR-655-3p in OSCC tissues (Fig. 4C). At the same time, miR-655-3p mimics reduced FZD4 expression, while miR-655-3p inhibitors promoted FZD4 expression in SCC-4 cells (Fig. 4 and E). Besides that, we also found that lncRNA SNHG5 was positively correlated with the expression of FZD4 in OSCC tissues (Fig. 4F). These results indicate that FZD4 is a direct target of miR-655-3p.
FZD4 regulates OSCC development by interacting with SNHG5/miR-655-3p axis. To elucidate the regulatory mechanism of 1ncRNA SNHG5, miR-655-3p and FZD4, the SNHG5 vector or miR-655-3p inhibitor was transfected into SCC-4 cells with FZD4 siRNA (si-FZD4). First, upregulation of FZD4 was examined in OSCC tissues and cells (Fig. 5A and B). And we found that si-FZD4 reduced its expression in SCC-4 cells. However, upregulation of SNHG5 or downregulation of miR-655-3p restored this decrease in FZD4 expression (Fig. 5C and D). Functionally, FZD4 silencing inhibited $\mathrm{SCC}-4$ cell proliferation. The SNHG5 vector or miR-655-3p inhibitor weakened the inhibitory effect of si-FZD4 on cell proliferation in SCC-4 cells (Fig. 5E). Moreover, knockdown of FZD4 suppressed the migration and invasion of SCC-4 cells. And upregulation of SNHG5 or downregulation of miR-655-3p eliminated the inhibitory effect of si-FZD4 on SCC-4 cell migration and invasion (Fig. 5F and G). Therefore, FZD4 promotes the progression of OSCC by interacting with the SNHG5/miR-655-3p axis. 

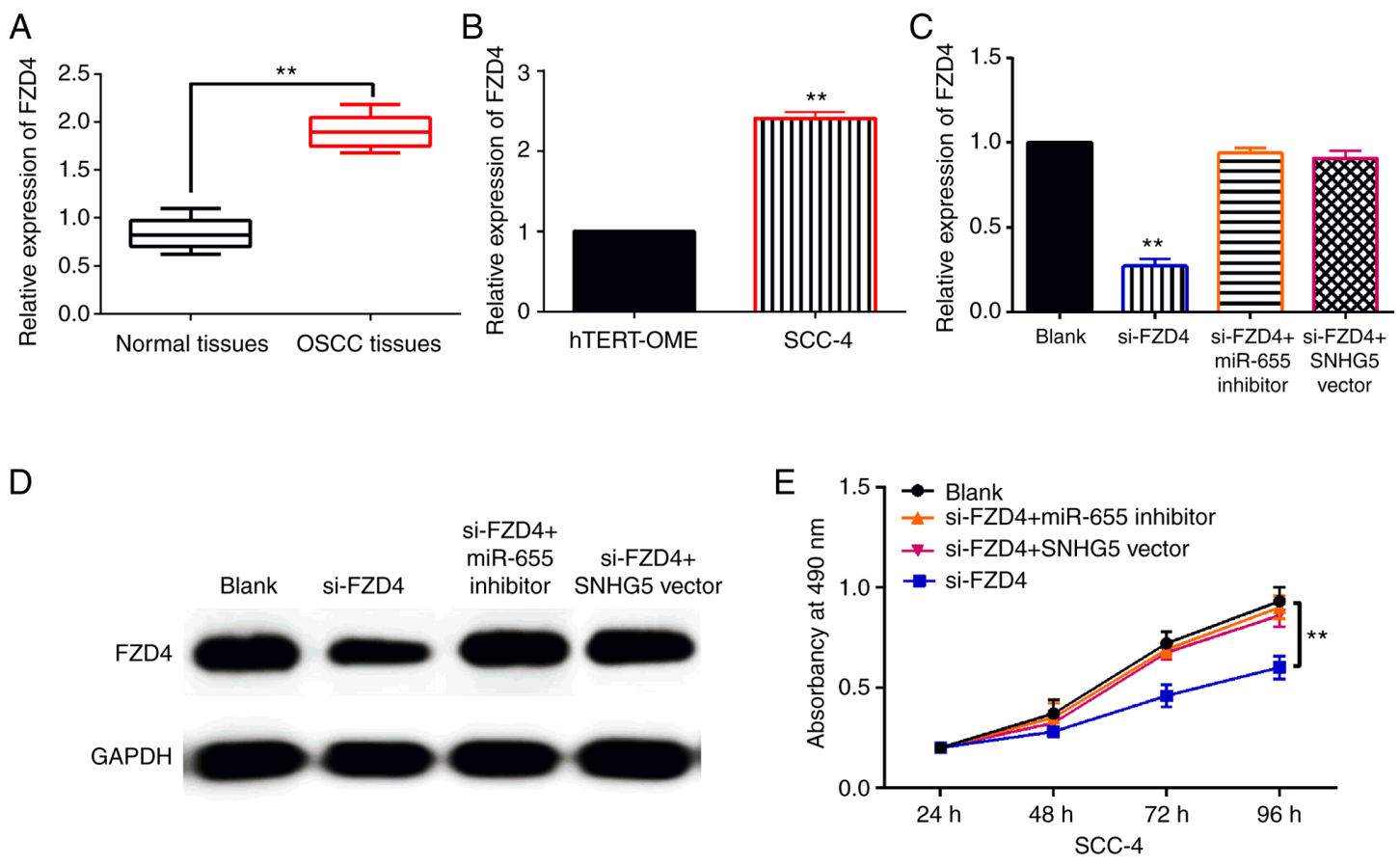

$\mathrm{F}$
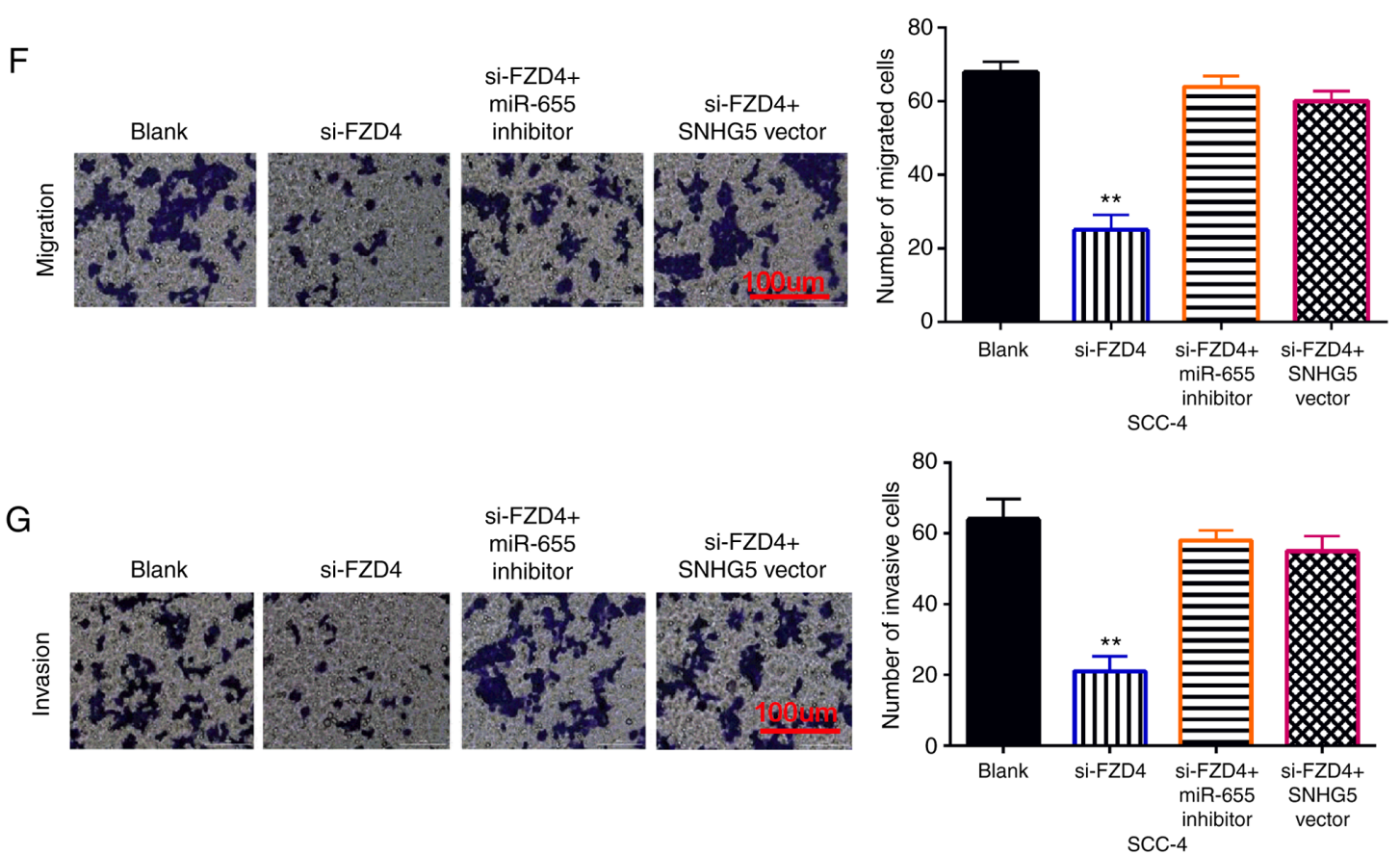

Figure 5. FZD4 regulates OSCC development by interacting with SNHG5/miR-655-3p axis. (A) The mRNA FZD4 expression in OSCC tissues (B) FZD4 expression in SCC-4 and hTERT-OME cells (C and D) FZD4 expression in SCC-4 cells with si-FZD4, si-FZD4+miR-655-3p inhibitor or si-FZD4+SNHG5 vector (E) Cell proliferation in SCC-4 cells with si-FZD4, si-FZD4+miR-655-3p inhibitor or si-FZD4+SNHG5 vector (F and G) Cell migration and invasion in SCC-4 cells with si-FZD4, si-FZD4+miR-655-3p inhibitor or si-FZD4+SNHG5 vector ${ }^{* *} \mathrm{P}<0.01$. OSCC, oral squamous cell carcinoma.

\section{Discussion}

Recently, the important roles of lncRNAs have been widely investigated in the progression of OSCC. Previous studies have indicated shown that IncRNA can play an inhibitory or carcinogenic effect in OSCC $(20,21)$. In this study, upregulation of lncRNA SNHG5 was detected in OSCC. And upregulation of lncRNA SNHG5 promoted cell proliferation, invasion, and migration in OSCC. Consistent with our results, SNHG5 was also upregulated in breast cancer and glioma $(22,23)$. Functionally, SNHG5 was found to promote the survival of colorectal cancer cells (24). In addition, SNHG5 promoted cell proliferation, invasion, and migration in human hepatocellular carcinoma (25). The same effect of SNHG5 on OSCC progression was also found in this study, but previous studies have not yet reported. All these findings suggest that lncRNA SNHG5 acts as a tumor promoter in the tumorigenesis of OSCC.

As we all know, lncRNA can act as a 'sponges' of miRNA by preventing miRNA from binding to a specific target. lncRNA SNHG5 has been reported to promote the progression of osteosarcoma and melanoma through sponging miR-26a-5p and miR-212-3p (26,27). Here, SNHG5 was 
identified as a sponge of miR-655-3p. In addition, downregulation of miR-655-3p was found in OSCC. And overexpression of miR-655-3p restrained the proliferation, invasion, and migration of OSCC cells. Reduced expression of miR-655-3p was also found in esophageal squamous cell carcinoma and triple-negative breast cancer $(13,28)$. In addition, miR-655-3p inhibited the proliferation and migration of ovarian cancer cells (29). It was also found that overexpression of miR-655 suppressed cell invasion (30). These findings are similar to our results, indicating that miR-655 plays an inhibitory role in OSCC.

Previous studies have shown that miRNAs are involved in tumor development by regulating gene expression. It has been reported that ADAM10, ZEB1 and TGFBR2 are direct targets of miR-655-3p (31,32). In our study, FZD4 was confirmed as a direct target of miR-655-3p. Moreover, FZD4 was upregulated in OSCC, and knockdown of FZD4 inhibited OSCC tumorigenesis. The same effect of FZD4 has also been found in non-small-cell lung cancer (33). Besides, it has been found that miR-505 acted as a tumor suppressor in cervical carcinoma by inversely regulating FZD4 (34). Here, we also found that miR-655-3p restrained the development of OSCC by downregulating FZD4. And lncRNA SNHG5 was found to promote the occurrence of OSCC by upregulating FZD4. Similarly, lncRNA DIX6-AS1 has been reported to promote the tumorigenesis of pancreatic cancer by regulating the miR-497-5p/FZD4 pathway (35). In conclusion, IncRNA SNHG5 promotes OSCC cell proliferation and metastasis by mediating the miR-655-3p/FZD4 axis. However, there are some limitations in this study, such as the absence of in vivo experiment. This study only explored the regulatory mechanism of lncRNA SNHG5 in vitro. The verification of our conclusion in vivo is still needed to be done in the future.

In conclusion, IncRNA SNHG5 promotes cell proliferation, migration and invasion in OSCC. In addition, lncRNA SNHG5 accelerates the progression of OSCC by downregulating miR-655-3p and upregulating FZD4. However, there is still an unknown about the regulatory mechanism of mechanism in OSCC. Therefore, further research will be conducted to clarify the functional mechanism of lncRNA SNHG5 in OSCC.

\section{Acknowledgements}

Not applicable.

\section{Funding}

No funding was received.

\section{Availability of data and materials}

The datasets used and/or analyzed in the present study are available from the corresponding author upon reasonable request.

\section{Authors' contributions}

LY and LH were responsible for the conception or design of the work. LY, XS and JZ contributed to the acquisition, analysis, or interpretation of data for the work. LY and XS provided the tissue samples. LH helped in the follow-up of the patients. JZ helped in reviewing the histopathology slides. JZ is the guarantor of the article. All authors read and approved the final manuscript.

\section{Ethics approval and consent to participate}

The study was approved by Ethical Committee of Peking Union Medical College Hospital and conducted in accordance with the ethical standards.

\section{Patient consent for publication}

Not applicable.

\section{Competing interests}

The authors declare that they have no competing interests.

\section{References}

1. Chi AC, Day TA and Neville BW: Oral cavity and oropharyngeal squamous cell carcinoma-an update. CA Cancer J Clin 65: 401-421, 2015.

2. Zhang SK, Zheng R, Chen Q, Zhang S, Sun X and Chen W: Oral cancer incidence and mortality in China, 2011. Chin J Cancer Res 27: 44-51, 2015.

3. Brands MT, Brennan PA, Verbeek ALM, Merkx MAW and Geurts SME: Follow-up after curative treatment for oral squamous cell carcinoma. A critical appraisal of the guidelines and a review of the literature. Eur J Surg Oncol 44: 559-565, 2018.

4. Morra F, Merolla F, Picardi I, Russo D, Ilardi G, Varricchio S, Liotti F, Pacelli R, Palazzo L, Mascolo M, et al: CAF-1 subunits levels suggest combined treatments with PARP-inhibitors and ionizing radiation in advanced HNSCC. Cancers (Basel) 11: $1582,2019$.

5. Sasahira T and Kirita T: Hallmarks of cancer-related newly prognostic factors of oral squamous cell carcinoma. Int J Mol Sci 19: 2413, 2018.

6. Bartonicek N, Maag JL and Dinger ME: Long noncoding RNAs in cancer: Mechanisms of action and technological advancements. Mol Cancer 15: 43, 2016.

7. Peng Z, Zhang C and Duan C: Functions and mechanisms of long noncoding RNAs in lung cancer. Onco Targets Ther 9: $4411-4424,2016$.

8. Jin Z, Jiang S, Jian S and Shang Z: Long noncoding RNA MORT overexpression inhibits cancer cell proliferation in oral squamous cell carcinoma by downregulating ROCK1. J Cell Biochem, Feb 252019 (Epub ahead of print). doi: 10.1002/jcb.28449.

9. Yang Y, Chen D, Liu H and Yang K: Increased expression of lncRNA CASC9 promotes tumor progression by suppressing autophagy-mediated cell apoptosis via the AKT/mTOR pathway in oral squamous cell carcinoma. Cell Death Dis 10: 41, 2019.

10. Zhao L, Guo H, Zhou B, Feng J, Li Y, Han T, Liu L, Li L, Zhang S, Liu Y, et al: Long non-coding RNA SNHG5 suppresses gastric cancer progression by trapping MTA2 in the cytosol. Oncogene 35: 5770-5780, 2016.

11. Hu X, Hong Y and Shang C: Knockdown of long non-coding RNA SNHG5 inhibits malignant cellular phenotypes of glioma via Wnt/CTNNB1 signaling pathway. J Cancer 10: 1333-1340, 2019.

12. Zhang M,Li Q, Pan Y, Wang H, Liu G and Yin H: MicroRNA-655 attenuates the malignant biological behaviours of retinoblastoma cells by directly targeting PAX6 and suppressing the ERK and p38 MAPK signalling pathways. Oncol Rep 39: 2040-2050, 2018.

13. Kiuchi J, Komatsu S, Imamura T, Nishibeppu K, Shoda K, Arita T, Kosuga T, Konishi H, Shiozaki A, Okamoto K, et al: Low levels of tumour suppressor miR-655 in plasma contribute to lymphatic progression and poor outcomes in oesophageal squamous cell carcinoma. Mol Cancer 18: 2, 2019. 
14. Gajera M, Desai N, Suzuki A, Li A, Zhang M, Jun G, Jia P, Zhao Z and Iwata J: MicroRNA-655-3p and microRNA-497-5p inhibit cell proliferation in cultured human lip cells through the regulation of genes related to human cleft lip. BMC Med Genomics 12: 70, 2019.

15. Wang W, Cao R, Su W, Li Y and Yan H: miR-655-3p inhibits cell migration and invasion by targeting pituitary tumor-transforming 1 in non-small cell lung cancer. Biosci Biotechnol Biochem 83: 1703-1708, 2019.

16. Lin J, Zandi R, Shao R, Gu J, Ye Y, Wang J, Zhao Y, Pertsemlidis A, Wistuba II, Wu X, et al: A miR-SNP biomarker linked to an increased lung cancer survival by miRNA-mediated down-regulation of FZD4 expression and Wnt signaling. Sci Rep 7: 9029, 2017.

17. Gupta S, Iljin K, Sara H, Mpindi JP, Mirtti T, Vainio P, Rantala J, Alanen K, Nees M and Kallioniemi O: FZD4 as a mediator of ERG oncogene-induced WNT signaling and epithelial-to-mesenchymal transition in human prostate cancer cells. Cancer Res 70 : 6735-6745, 2010

18. Chen L, Long Y, Han Z, Yuan Z, Liu W, Yang F, Li T, Shu L and Zhong Y: MicroRNA-101 inhibits cell migration and invasion in bladder cancer via targeting FZD4. Exp Ther Med 17: 1476-1485, 2019.

19. Wang Y, Zhang W, Wang Y and Wang S: HOXD-AS1 promotes cell proliferation, migration and invasion through miR-608/FZD4 axis in ovarian cancer. Am J Cancer Res 8: 170-182, 2018.

20. Tan J, Xiang L and Xu G: IncRNA MEG3 suppresses migration and promotes apoptosis by sponging miR-548d-3p to modulate JAK-STAT pathway in oral squamous cell carcinoma. IUBMB Life 71: 882-890, 2019.

21. Li B, Wang W, Miao S, Li G, Lv Y, Xiang C and Pei R HOXA11-AS promotes the progression of oral squamous cell carcinoma by targeting the miR-518a-3p/PDK1 axis. Cancer Cell Int 19: 140, 2019.

22. Chi JR, Yu ZH, Liu BW, Zhang D, Ge J, Yu Y and Cao XC: SNHG5 promotes breast cancer proliferation by sponging the miR-154-5p/PCNA axis. Mol Ther Nucleic Acids 17: 138-149, 2019

23. Li X, Liu L, Luo Y, Cui S, Chen W, Zeng A, Shi Y and Luo L: Long non-coding RNA SNHG5 promotes glioma progression via miR-205/E2F3 axis. Biosci Rep 39: BSR20190668, 2019.

24. Damas ND, Marcatti M, Come C, Christensen LL, Nielsen MM, Baumgartner R, Gylling HM, Maglieri G, Rundsten CF Seemann SE, et al: SNHG5 promotes colorectal cancer cell survival by counteracting STAU1-mediated mRNA destabilization. Nat Commun 7: 13875, 2016.

25. Li Y, Guo D, Zhao Y, Ren M, Lu G, Wang Y, Zhang J, Mi C, $\mathrm{He} \mathrm{S}$ and $\mathrm{Lu} \mathrm{X}$ : Long non-coding RNA SNHG5 promotes human hepatocellular carcinoma progression by regulating miR-26a-5p/GSK3 $\beta$ signal pathway. Cell Death Dis 9: 888, 2018
26. Ju C, Zhou R, Sun J, Zhang F, Tang X, Chen KK, Zhao J, Lan X, Lin S, Zhang Z and Lv XB: IncRNA SNHG5 promotes the progression of osteosarcoma by sponging the miR-212-3p/SGK3 axis. Cancer Cell Int 18: 141, 2018.

27. Gao J, Zeng K, Liu Y, Gao L and Liu L: lncRNA SNHG5 promotes growth and invasion in melanoma by regulating the miR-26a-5p/TRPC3 pathway. Onco Targets Ther 12: 169-179, 2018.

28. Lv ZD, Kong B, Liu XP, Jin LY, Dong Q, Li FN and Wang HB: miR-655 suppresses epithelial-to-mesenchymal transition by targeting Prrx1 in triple-negative breast cancer. J Cell Mol Med 20: 864-873, 2016.

29. Zha JF and Chen DX: miR-655-3p inhibited proliferation and migration of ovarian cancer cells by targeting RAB1A. Eur Rev Med Pharmacol Sci 23: 3627-3634, 2019.

30. Wang Y, Zang W, Du Y, Ma Y, Li M, Li P, Chen X, Wang T, Dong Z and Zhao G: Mir-655 up-regulation suppresses cell invasion by targeting pituitary tumor-transforming gene- 1 in esophageal squamous cell carcinoma. J Transl Med 11: 301, 2013.

31. Wu G, Zheng K, Xia S, Wang Y, Meng X, Qin X and Cheng Y: MicroRNA-655-3p functions as a tumor suppressor by regulating ADAM10 and $\beta$-catenin pathway in hepatocellular carcinoma. J Exp Clin Cancer Res 35: 89, 2016.

32. Harazono Y, Muramatsu T, Endo H, Uzawa N, Kawano T, Harada K, Inazawa $J$ and Kozaki $K$ : miR-655 is an EMT-suppressive microRNA targeting ZEB1 and TGFBR2. PLoS One 8: e62757, 2013.

33. Yang Y, Sun Y, Wu Y, Tang D, Ding X, Xu W, Su B and Gao W: Downregulation of miR-3127-5p promotes epithelial-mesenchymal transition via FZD4 regulation of Wnt/ $\beta$-catenin signaling in non-small-cell lung cancer. Mol Carcinog 57: 842-853, 2018.

34. Ma C, Xu B, Husaiyin S, Wang L, Wusainahong K, Ma J, Zhu K and Niyazi M: MicroRNA-505 predicts prognosis and acts as tumor inhibitor in cervical carcinoma with inverse association with FZD4. Biomed Pharmacother 92: 586-594, 2017.

35. Yang J, Ye Z, Mei D, Gu H and Zhang J: Long noncoding RNA DLX6-AS1 promotes tumorigenesis by modulating $\mathrm{miR}-497-5 \mathrm{p} / \mathrm{FZD} 4 / \mathrm{FZD} 6 / \mathrm{Wnt} / \beta$-catenin pathway in pancreatic cancer. Cancer Manag Res 11: 4209-4221, 2019.

(i) (2) This work is licensed under a Creative Commons Attribution-NonCommercial-NoDerivatives 4.0 International (CC BY-NC-ND 4.0) License. 